\title{
O Ventrículo Direito e a Hipertensão Arterial. Aspectos Ecocardiográficos
}

\author{
Ricardo Pereira Silva, Celso Amodeo, José Antônio Franchini Ramires \\ Fortaleza, CE - São Paulo, SP
}

O exame ecocardiográfico do ventrículo direito tem muitas limitações. Grande parte da câmara situa-se atrás do esterno, possui formato irregular, paredes trabeculadas e localização variável dentro do tórax, na dependência da posição do paciente. Apesar destes problemas, o ecocardiograma pode fornecer informações úteis sobre o ventrículo direito, incluindo a determinação de suas dimensões ${ }^{1}$.

A determinação do tamanho do ventrículo direito pode ser feita qualitativa ou quantitativamente. Na primeira forma, compara-se o seu tamanho ao do ventrículo esquerdo, utilizando-se o corte apical de quatro câmaras. Para a avaliação quantitativa, utiliza-se o modo $\mathrm{M}$ ou o bidimensional. Utilizando-se esta segunda modalidade, pode-se medir o volume do ventrículo direito através de várias técnicas, dentre as quais a regra de Simpson. Neste método, o ventrículo direito é dividido numa série de fatias de igual espessura. $\mathrm{O}$ volume de cada fatia é calculado com a fórmula: volume = área $\mathrm{x}$ altura. $\mathrm{O}$ volume da câmara é obtido pela soma dos volumes das fatias individuais. Alternativamente, cada fatia pode ser representada por uma figura geométrica simples e a fórmula volumétrica para esta figura é usada para calcular o volume da fatia. No entanto, até o momento, nenhuma técnica para cálculo de volume foi introduzida nos exames de rotina $^{2}$.

A sobrecarga de pressão do ventrículo direito é detectada, primariamente, pela hipertrofia da sua parede livre, podendo também ser encontrada hipertrofia do septo interventricular. A sobrecarga de pressão também pode levar a dilatação desta câmara ou a distorção do septo interventricular durante a sístole ${ }^{2}$.

Hospital Universitário Walter Cantídio - UFCE - Fortaleza

Correspondência: Ricardo Pereira Silva - Rua Dr. José Lourenço, 2275/102

60115-282 Fortaleza, CE - E-mail: ricardop@ultranet.com.br

Recebido para publicação em 12/4/01

Aceito em 5/9/01

\section{A hipertrofia do ventrículo esquerdo na hiper- tensão arterial}

Em alguns aspectos, a hipertrofia do ventrículo esquerdo em hipertensos parece ser um paradoxo. Ela atenua o efeito da sobrecarga de pressão que a hipertensão promove sobre a parede do ventrículo esquerdo e aumenta a função contrátil global do coração. Por outro lado, a sua presença está relacionada com maior aumento de risco cardiovascular expresso por insuficiência cardíaca e morte súbita ${ }^{3}$.

A hipertrofia ventricular esquerda nos hipertensos parece ser, inicialmente, um mecanismo compensatório ao aumento da pós-carga, mas está associada a alterações estruturais e funcionais do miocárdio. Alguns autores relacionam a massa do ventrículo esquerdo com a pressão arterial medida clinicamente, confirmado em estudos subseqüentes ${ }^{4,5}$. A fraca correlação entre a massa do ventrículo esquerdo e a pressão arterial em grandes séries de pacientes levou alguns investigadores a propor que estímulos nãohemodinâmicos poderiam ter papel fundamental na patogênese da hipertrofia ventricular esquerda na hipertensão arterial, contudo, tem sido sugerido que a importância dos fatores hemodinâmicos na determinação da massa do ventrículo esquerdo em hipertensos foi subestimada por duas razões: 1) medidas casuais de pressão arterial freqüentemente não são representativas da sobrecarga pressórica imposta pela hipertensão arterial; 2) a sobrecarga hemodinâmica imposta ao coração não é devido apenas aos níveis de pressão arterial mas também à variabilidade na sobrecarga de volume, na complacência arterial e em outros fatores ${ }^{6-7}$.

Os estímulos não-hemodinâmicos para a hipertrofia ventricular esquerda na hipertensão arterial encontram-se, principalmente, no sistema nervoso autônomo e no sistema renina-angiotensina. $O$ conceito de que a atividade adrenérgica cardíaca modula a hipertrofia do miocárdio baseia-se no fato de que a administração de catecolamina induz um grau de hipertrofia ventricular esquerda desproporcional às alterações hemodinâmicas. Por outro lado, a atividade adrenérgica parece não ter papel independente na hipertrofia cardíaca. A ação direta da angiotensina II na hipertrofia do 
miocárdio tem sido sugerida pelos seguintes achados: localização preferencial de angiotensina marcada em zonas nucleares de miócitos vasculares e cardíacos; estímulo da síntese protéica no miocárdio depois da infusão de angiotensina; redução na massa do ventrículo esquerdo em pacientes que usam inibidores da enzima de conversão ${ }^{8}$.

O gene da enzima de conversão da angiotensina, em sua região não-codificante (intron 16), tem duas formas alélicas, dependendo da presença ("I" de inserção) ou ausência ("D" de deleção) de uma sequiência de 287 pares de bases de DNA. O alelo D está associado a diferentes doenças cardiovasculares. Os níveis circulantes e cardíacos da enzima de conversão estão aumentados no genótipo DD. O gene do angiotensinogênio tem muitas variantes. A variante metionina-tironina na posição 235 está associada à hipertensão arterial, no entanto existe controvérsia entre o polimorfismo I/D da enzima de conversão e a hipertrofia do ventrículo esquerdo ${ }^{9}$.

O envolvimento potencial do sistema renina angiotensina cerebral na hipertensão arterial induzida por doses subpressoras de angiotensina II foi testado em ratos transgênicos desenvolvidos com permanente inibição da síntese de angiotensinogênio. A pressão arterial sistólica foi significativamente mais baixa nos ratos transgênicos do que nos ratos-controle. $\mathrm{O}$ aumento no índice de massa cardíaca foi evidente apenas nos ratos-controle. Os níveis de RNAm do peptídeo natriurético atrial e o colágeno do ventrículo esquerdo estiveram aumentados nos dois grupos de ratos, como conseqüência da infusão de angiotensina II. Assim, a expressão destes genes, no ventrículo esquerdo, parece estar diretamente estimulada pela angiotensina II, contudo os efeitos hipertensivo e hipertrófico da dose subpressora de angiotensina II são, pelo menos em parte, mediados pelo sistema renina angiotensina cerebral ${ }^{10}$.

\section{Alterações do ventrículo direito na vigência de hipertrofia do ventrículo esquerdo}

Além dos efeitos da hipertensão arterial sobre o ventrículo esquerdo, alguns estudos passaram a demonstrar que a hipertensão arterial sistêmica também causava repercussão no ventrículo direito, observando-se alterações na circulação pulmonar e, em conseqüência, anormalidade da espessura e da função dessa câmara ${ }^{11}$.

Quando uma sobrecarga venosa sistêmica ocorre em um indivíduo hipertenso, presume-se que o ventrículo direito não foi capaz de suportar a sobrecarga imposta pela insuficiência do ventrículo esquerdo e, conseqüentemente, dilatou-se e entrou em falência. No entanto, em 1908, Bernheim chamava a atenção para um paradoxo no qual se observava a presença de redução ao invés de dilatação do ventrículo direito em pacientes que haviam falecido com a sintomatologia clássica de insuficiência cardíaca direita. A conclusão a que chegou Bernheim foi que a hipertrofia e a dilatação do ventrículo esquerdo haviam sido responsáveis por uma invasão do septo interventricular na cavidade ventricular direita. A redução do ventrículo direito poderia ser considerada, quando um paciente com hipertrofia do ventrículo esquerdo apresentava sintomas de insuficiência cardíaca direita na ausência de sinais de insuficiência cardíaca esquerda, como dispnéia ou congestão pulmonar ${ }^{12}$.

Em 1949, foi publicado o relato de quatro casos que exemplificariam a síndrome de Bernheim, sendo dois pacientes com estenose aórtica e dois pacientes com hipertensão arterial. East e Bain comentavam que, na síndrome de Bernheim, os sintomas sugestivos de insuficiência ventricular direita surgiam precocemente e podiam ser transitórios e que, por não atingir a circulação pulmonar, os pacientes não apresentavam ortopnéia. O diagnóstico clínico seria confirmado na autópsia pela aparência normal dos pulmões com hipertrofia do septo interventricular ${ }^{13}$.

No entanto, em 1955, publicou-se o relato de caso de um homem hipertenso com doença coronariana que evoluiu com insuficiência cardíaca sistêmica sem dispnéia, ortopnéia ou congestão pulmonar. E, na necropsia, o septo estava desviado para a direita, estenosando a cavidade do ventrículo direito ${ }^{14}$. Esse caso preencheria os critérios clínicos e patológicos da síndrome de Bernheim, no entanto o estudo hemodinâmico revelou achados convencionais de insuficiência ventricular esquerda e direita e hipertensão pulmonar. Segundo os autores, o substrato patológico da síndrome de Bernheim estaria baseado numa concepção equivocada, pois o septo interventricular, mesmo em indivíduos normotensos, encontra-se desviado para a direita, invadindo a cavidade crescêntica do ventrículo direito; esta relação está exacerbada nos corações com hipertrofia do ventrículo esquerdo, independente do quadro clínico. Desta maneira, os casos relatados como síndrome de Bernheim representariam exemplos de insuficiência cardíaca, nos quais a dispnéia e a congestão pulmonar eram pouco significativas.

A hipertrofia ventricular direita pode ocorrer em pacientes com hipertrofia ventricular esquerda e acreditava-se que ela fosse secundária à hipertensão pulmonar. Entretanto, Gottdiener e cols. compararam um grupo de pacientes com sobrecarga pressórica do ventrículo esquerdo, constituído por hipertensos ou portadores de estenose aórtica, com um grupo controle constituído por indivíduos normotensos ou com miocardiopatia dilatada, e encontraram que a espessura do ventrículo direito era maior no primeiro que no segundo grupo. A hipertrofia do ventrículo direito esteve presente em $80 \%$ dos pacientes hipertensos e em $63 \%$ dos pacientes com estenose aórtica. A magnitude da hipertrofia do ventrículo direito esteve correlacionada linearmente com a hipertrofia do ventrículo esquerdo, mas não houve associação com hipertensão pulmonar, o que levou os autores a concluir que fatores indefinidos, diferentes da sobrecarga de pressão, podem funcionar como estímulo para o aumento da espessura das paredes ventriculares ${ }^{15}$. A ausência de correlação entre a espessura do ventrículo direito e hipertensão pulmonar também foi ressaltada por outros autores ${ }^{16}$.

A interdependência morfológica e funcional entre os dois ventrículos poderia ser explicada por três mecanismos: 1) aumento da pressão diastólica final do ventrículo direito em resposta ao aumento do volume do ventrículo esquer- 
do; 2) transmissão mecânica da tensão sofrida pelo ventrículo esquerdo ao ventrículo direito através das fibras musculares comuns aos dois ventrículos; 3 ) fatores humorais, como as catecolaminas, poderiam mediar a hipertrofia dos ventrículos como resposta à sobrecarga pressórica de um dos dois ventrículos ${ }^{15}$.

Os ventrículos guardam entre si íntima relação anatômica. As fibras da via de entrada do ventrículo direito estão orientadas como as fibras do ventrículo esquerdo. Elas se originam do triângulo esquelético posterior e circundam o orifício da valva tricúspide. A via de saída do ventrículo direito tem um desenvolvimento embriológico peculiar, nascendo das fibras circunvasculares. Asfibras da via de saída do ventrículo direito se originam do triângulo esquelético anterior, circundam a região do cone e emergem próximo à sua origem, quando se misturam com as fibras do ventrículo esquerdo ${ }^{17}$.

A interdependência funcional entre os dois ventrículos já havia sido sugerida. Olivari e cols. procederam à monitorização hemodinâmica com cateter de Swan-Ganz e medida invasiva de pressão arterial em dois grupos de hipertensos com hipertrofia ventricular esquerda (o primeiro sem sinais de strain ao eletrocardiograma e o segundo com sinais de strain) e em um grupo de indivíduos normotensos. Os autores encontraram aumento da resistência pulmonar no primeiro grupo, que se encontrava ainda mais aumentada no segundo grupo, o que levou ao aumento da pressão de artéria pulmonar nos dois grupos. No primeiro grupo, observou-se melhor função ventricular esquerda e direita, enquanto que, no segundo grupo, foi observada pior função ventricular esquerda e direita, quando comparada ao terceiro grupo (controle) ${ }^{18}$.

Bloomfield e cols. ${ }^{19}$ relataram a presença de valores normais de pressão arterial pulmonar em hipertensos, demonstrando que o aumento da resistência arteriolar sistêmica não teria influência na circulação pulmonar .

No entanto, quatro décadas após, Ramires submeteu pacientes hipertensos a exercício físico e observou aumento da pressão do átrio direito na maioria dos pacientes, associado a aumento do débito cardíaco. Esse achado refletiria um aumento da pré-carga, concorrendo paraum melhor desempenho do ventrículo direito. Esse dado foi confirmado, quando se analisou o trabalho do ventrículo direito, sugerindo que a melhora da função ventricular direita na hipertensão arterial, durante o exercício físico, dependeria do retorno venoso.

Nesse mesmo estudo, a resistência arterial pulmonar já se encontrava aumentada em repouso na maioria dos pacientes, levando a crer que, na hipertensão arterial, existem fatores que modificam o comportamento da circulação pulmonar ${ }^{20}$.

Outros autores confirmaram esses achados e demonstraram que a circulação pulmonar dos pacientes hipertensos pode sofrer influências, independentes da falência ventricular esquerda e, algumas vezes, sua resposta é diferente da que ocorre na circulação sistêmica ${ }^{21,22}$.

Oliveira estudou hipertensos submetidos a exercício físico e os dividiu em dois grupos, segundo o índice cardíaco (grupo I, com índice cardíaco $\geq 2,5 \mathrm{ml} / \mathrm{min} / \mathrm{m}^{2}$, e grupo II, com índice cardíaco $<2,5 \mathrm{ml} / \mathrm{min} / \mathrm{m}^{2}$ ) e encontrou comporta- mento semelhante dos dois grupos no que se refere à pressão arterial sistólica, diastólica e média, à pressão do átrio direito, à pressão capilar pulmonar e à frequiência cardíaca. Comportamento diferente foi identificado no índice sistólico, na resistência arterial pulmonar e na resistência vascular sistêmica. A redução da resistência arterial pulmonar nos hipertensos com disfunção ventricular esquerda submetidos a exercício físico, quando comparada à fase de repouso, sugere a influência da insuficiência ventricular esquerda na resistência arterial pulmonar ${ }^{23}$.

Em outro estudo, no qual hipertensos foram submetidos a exercício físico, os pacientes também foram divididos em dois grupos, desta vez segundo o nível de pressão sistólica em artéria pulmonar atingido com esforço. Observou-se redução da resistência arterial pulmonar com esforço, que seria uma forma de se manter o desempenho do ventrículo direito ${ }^{24}$.

Com o objetivo de determinar a severidade da doença miocárdica em relação à hipertrofia dos miócitos e da fibrose intersticial no miocárdio dos ventrículos, pesquisadores japoneses realizaram biópsia endomiocárdica biventricular em um grupo de pacientes com hipertensão arterial essencial, divididos, segundo o grau de hipertensão, como leve, moderada ou severa. Foi encontrado aumento da largura dos miócitos tanto de ventrículo direito como de ventrículo esquerdo, à medida que o grau de hipertensão era mais severo. O mesmo comportamento observou-se em relação à fibrose intersticial, que aumentou de acordo com o grau de severidade da hipertensão. Foi observado ainda que a hipertrofia dos miócitos e o aumento da fibrose intersticial nos ventrículos progridem de maneira proporcional também às alterações do eletrocardiograma ${ }^{25}$.

\section{Determinação da hipertrofia do ventrículo di- reito pelo ecocardiograma}

Através do ecocardiograma, é possível se medir a espessura da parede livre do ventrículo direito e tentar observar se este ventrículo sofre as conseqüências da hipertensão arterial.

Prakash e cols., ao determinar como espessura normal do ventrículo direito à necropsia um valor inferior a $2 \mathrm{~mm}$ e como hipertrofia do ventrículo direito uma espessura maior ou igual a $5 \mathrm{~mm}$, encontraram boa correlação entre a medida da espessura do ventrículo direito em sístole e diástole através do ecocardiograma modo $\mathrm{M}$ e à necropsia, tanto para indivíduos com espessura normal quanto para aqueles com espessura aumentada ${ }^{26}$. Os mesmos autores, em outra publicação do mesmo ano, encontraram uma correlação de 0,82 entre a espessura do ventrículo direito pelo ecocardiograma e pela necropsia ${ }^{27}$.

Também utilizando ecocardiograma modo M, Nuneze cols. mediram a espessura da parede livre do ventrículo direito de pacientes com hipertrofia do ventrículo esquerdo e compararam com hipertensos sem hipertrofia do ventrículo esquerdo e com um grupo de indivíduos normotensos. Foi observado que a espessura da parede livre do ventrículo direito nos hipertensos com hipertrofia do ventrículo es- 
querdo esteve aumentada quase duas vezes em relação aos normotensos. Por outro lado, os hipertensos sem hipertrofia do ventrículo esquerdo demonstravam apenas tendência ao aumento da espessura do ventrículo direito, sem diferença estatisticamente significante. Foi observado, ainda, que o índice de esvaziamento do átrio esquerdo esteve reduzido em todos os pacientes hipertensos, independente da presença de hipertrofia do ventrículo esquerdo, sugerindo envolvimento funcional precoce do ventrículo esquerdo na hipertensão $\operatorname{arterial}^{28}$.

Através do ecocardiograma bidimensional, observouse que a média da espessura da parede livre do ventrículo direito em indivíduos normotensos não ultrapassa $4 \mathrm{~mm}$, no entanto, valores individuais podem chegar até $7 \mathrm{~mm}^{1}$.

Baseados em outros parâmetros, Cacho e cols. estudaram a utilidade do ecocardiograma bidimensional na detecção da hipertrofia ventricular direita. Nesse trabalho, a hipertrofia do ventrículo direito foi previamente estabelecida pela presença de pelo menos dois entre três critérios. Como critério de exame físico, adotou-se a hiperfonese do componente pulmonar da segunda bulha e/ou a elevação esternal. O critério fornecido pelo ecocardiograma modo $\mathrm{M}$ foi a espessura diastólica do ventrículo direito maior que $5 \mathrm{~mm}$, espessura sistólica maior que $9 \mathrm{~mm}$ ou ainda diâmetro diastólico maior que $28 \mathrm{~mm}$. O terceiro critério foi a evidência de sobrecarga ventricular direita ao eletrocardiograma. Quando foram realizadas as medidas pelo ecocardiograma bidimensional, no corte apical de quatro câmaras, e comparado um grupo de pacientes com hipertrofia do ventrículo direito pelos critérios expostos com um grupo de indivíduos normotensos, observou-se diferença estatisticamente significante nos diâmetros longitudinal e transversal e área do átrio direito e no diâmetro longitudinal e área do ventrículo direito ${ }^{29}$.

Ainda no que se refere à determinação da hipertrofia do ventrículo direito pelo ecocardiograma, Devereux e cols. mediram o diâmetro diastólico e a espessura do ventrículo direito em adultos sem cardiopatia congênita que, ao falecer, foram encaminhados para a necropsia num prazo inferior a 77 dias após a realização do exame. A medida da espessura do ventrículo direito ao ecocardiograma teve correlação razoável com a medida da espessura na necropsia $(\mathrm{r}=0,59)$, mas não teve correlação com o peso do ventrículo direito na necropsia, enquanto que a medida do diâmetro diastólico do ventrículo direito teve boa correlação $(\mathrm{r}=0,69)$ com o peso da câmara na necropsia. Nesse estudo, um diâmetro diastólico superior a $30 \mathrm{~mm}$ detectou hipertrofia do ventrículo direito em adultos com sensibilidade de $67 \%$ e especificidade de $92 \%$. Os autores afirmam que a espessura do ventrículo direito ao ecocardiograma não guarda relação com seu peso, porque este se relaciona predominantemente com a dilatação da câmara ${ }^{30}$.

\section{Influência da hipertensão arterial na função dos ventrículos}

Para a manutenção da função adequada do ventrículo direito, é importante que a contração do septo interventricular esteja preservada ${ }^{31}$. Relacionando a função do ventrículo esquerdo, através da fração de ejeção, com a espessura do ventrículo direito, demonstrou-se que, em portadores de enfisema pulmonar, a hipertrofia do ventrículo direito é o mecanismo responsável pela manutenção da fração de ejeção do ventrículo esquerdo, o que é explicado pelo fato da pressão sistólica do ventrículo direito elevada empurrar o septo interventricular para a esquerda, diminuindo a sua curvatura. O deslocamento do septo é mais pronunciado no final da sístole que no final da diástole, causando uma inversão sistólica no ventrículo esquerdo. Esse auxílio do ventrículo direito na sístole pode ser responsável pela preservação da fração de ejeção do ventrículo esquerdo nos pacientes enfisematosos com hipertrofia do ventrículo direito ${ }^{32}$.

Outro questionamento que se vem fazendo em relação aos hipertensos refere-se à função de ambos os ventrículos. Ferlinz ${ }^{33}$ realizou cateterismo cardíaco em 20 indivíduos hipertensos e constatou aumento da pressão no átrio direito, aumento da pressão diastólica final do ventrículo direito, aumento da pressão em artéria pulmonar e em capilar pulmonar, débito cardíaco normal e queda da fração de ejeção do ventrículo direito. Nenhum dos pacientes apresentava evidência clínica, radiológica ou hemodinâmica de disfunção do ventrículo esquerdo, não podendo ser considerada, portanto, a causa do aumento de pressão nas câmaras direitas. Segundo o autor, a causa do aumento de pressão poderia ser a síndrome de Bernheim: a hipertrofia do septo interventricular invadiria o ventrículo direito a tal ponto, que haveria uma obstrução funcional dentro do ventrículo direito, levando ao aumento da pressão nas câmaras direitas e, posteriormente, à disfunção do ventrículo direito. No entanto, na síndrome de Bernheim, a circulação pulmonar permanece inalterada e o volume do ventrículo direito diminui, enquanto que, nesse trabalho, houve aumento da pressão em artéria pulmonar e aumento do volume do ventrículo direito.

Outra explicação para o aumento de pressão nas câmaras direitas seria o efeito de vários mecanismos humorais presentes na pequena circulação. Sabe-se que pacientes hipertensos têm aumento da atividade simpática com aumento da concentração plasmática de catecolaminas em repouso e aumento da angiotensina. Sabe-se ainda que a circulação pulmonar responde à noradrenalina e à angiotensina com vasoconstrição pulmonar e aumento da pressão de artéria pulmonar. Desta maneira, a hipertensão arterial exerceria influência no coração, como um todo, reforçando o conceito de que os ventrículos direito e esquerdo representam, fisiologicamente, uma unidade funcional única ${ }^{33}$.

Ao contrário do que sugeria Olivari e cols., que a função do ventrículo direito na hipertensão arterial podia ser predita com base no eletrocardiograma (hipertrofia do ventrículo esquerdo sem padrão de strain encontrada em indivíduos com função do ventrículo direito acima do normal e hipertrofia do ventrículo esquerdo com padrão de strain encontrada em indivíduos com disfunção do ventrículo direito) ${ }^{18}$, achados que não foram confirmados pelo estudo de Ferlinz ${ }^{33}$.

Cohn e cols. resumiram os mecanismos que poderiam explicar a disfunção do ventrículo direito nos hipertensos: 
1) aumento crônico da pressão em artéria pulmonar secundária à disfunção do ventrículo esquerdo. Contra este argumento, sabe-se que é pouco provável que o ventrículo direito entre em falência após exposição por curto tempo a uma discreta elevação da pressão em artéria pulmonar; 2) hipertrofia do septo interventricular invadindo o ventrículo direito e dificultando o seu enchimento (teoria da síndrome de Bernheim, em desuso); 3) a disfunção do ventrículo direito representaria uma capacidade intrínseca desta câmara de alterar suas características de esvaziamento com a finalidade de prevenir uma emissão de volume aumentado para o ventrículo esquerdo, que já está sobrecarregado ${ }^{34}$.

A sobrecarga de pressão e de volume do ventrículo direito tem efeitos contrários na fração de ejeção do ventrículo esquerdo. Foi demonstrado que, apesar de haver redução relativa do enchimento do ventrículo esquerdo na sobrecarga de pressão do ventrículo direito, a fração de ejeção do ventrículo esquerdo permanece preservada, enquanto que esta fração de ejeção se encontra deprimida nos pacientes com sobrecarga volumétrica do ventrículo direito ${ }^{35,36}$.

O enchimento e a função do ventrículo esquerdo podem ser secundariamente influenciados pela interdependência entre os dois lados do coração, criada pela sua disposição como bombas em série, bem como pela interação mecânica via septo interventricular, que é comum aos dois ventrículos, e pelas fibras miocárdicas circunferenciais que circundam ambas as câmaras ${ }^{35}$.

\section{$O$ efeito do tratamento anti-hipertensivo}

O impacto da regressão da hipertrofia ventricular esquerda na função sistólica e diastólica dos ventrículos permanecia controverso até pouco tempo, quando Habib e cols. estudaram um grupo de pacientes hipertensos com ecocardiograma, antes de iniciar tratamento anti-hipertensivo com nifedipina e após um, três, cinco e sete meses de tratamento. Foi observada regressão da massa do ventrículo esquerdo com um mês de tratamento, regredida mais ainda com três meses, permanecendo depois inalterada. Comportamento semelhante teve a dinâmica de enchimento dos dois ventrículos, que melhorou durante os três primeiros meses de tratamento, permanecendo depois inalterada. Também houve melhora na função sistólica do ventrículo esquerdo ${ }^{37}$.

O mesmo autor, em publicação anterior, já havia demonstrado que a dinâmica de enchimento do ventrículo direito está alterada na hipertensão arterial independente da massa do ventrículo esquerdo ou dos níveis de pressão arterial. Foi demonstrada uma fraca correlação da dinâmica de enchimento do ventrículo direito com a espessura do ventrículo direito e uma grande correlação com a dinâmica de enchimento do ventrículo esquerdo ${ }^{38}$.

Ainda no que concerne aos efeitos do tratamento antihipertensivo na estrutura e função dos ventrículos, Cuspidi e cols., utilizando captopril isoladamente e em associação com nifedipina, também demonstraram redução da massa do ventrículo esquerdo e da espessura do ventrículo direito, além de redução na onda $\mathrm{A} e$ aumento da onda $\mathrm{E}$ nos fluxos mitral e tricúspide ${ }^{11}$.
Outros investigadores, no entanto, encontraram resultados diferentes, utilizando os mesmos grupos de drogas anti-hipertensivas. Apesar de ter havido alterações hemodinâmicas e redução de massa do ventrículo esquerdo semelhantes entre os dois grupos de pacientes utilizando drogas anti-hipertensivas, a espessura do ventrículo direito aumentou nos pacientes que utilizaram bloqueadores dos canais de cálcio e não se alterou nos pacientes tratados com inibidores da enzima de conversão da angiotensina, demonstrando que bloqueadores dos canais de cálcio e inibidores da enzima de conversão da angiotensina podem produzir efeitos distintos no crescimento das células cardíacas ${ }^{39}$.

\section{Novas técnicas para a medida da massa do ventrículo direito}

Para verificar o possível envolvimento do ventrículo direito na hipertensão arterial, parece que a simples determinação da sua espessura é insuficiente e que o ideal seria determinar o valor da massa desta câmara.

A razão de se tentar uma nova técnica para medir a massa do ventrículo direito deve-se à natural dificuldade de visibilização desta câmara, que se encontra parcialmente atrás do esterno.

Outras técnicas foram utilizadas para este fim, como a fórmula de Simpson, sem resultados satisfatórios. A assimetria ventricular direita e a ausência de marcadores internos que pudessem orientar a localização dos planos de corte devem ter contribuído para que fosse encontrado baixo índice de correlação entre os volumes calculados por esse método e pela angiografia ${ }^{40,41}$.

Mais recentemente, foi publicado um método desenvolvido por Pontes Jr. para cálculo da massa da parede livre do ventrículo direito, através do ecocardiograma bidimensional, que procura superar as dificuldades inerentes à análise desta câmara. Esse método, denominado originalmente de método dos perímetros internos e validado em cães, baseia-se no formato da parede livre do ventrículo direito, que, após dissecada, apresenta-se como uma superfície plana de forma trapezoidal. A área desta superfície é calculada pela fórmula da área do trapézio. Para isso, utilizam-se três perímetros ecocardiográficos do ventrículo direito: o eixo menor da valva aórtica e do átrio esquerdo, o eixo menor ao nível médio ventricular e a posição apical de quatro câmaras. Cada um do perímetros representa as duas bases e a altura do trapézio, respectivamente. A área obtida é multiplicada pela espessura, resultando no volume da parede livre do ventrículo direito, que, por sua vez, é multiplicado pela massa específica do miocárdio, resultando na massa do miocárdio do ventrículo direito ${ }^{42}$.

Concluindo, achamos que este método utilizado para cálculo da massa do ventrículo direito veio preencher uma lacuna existente na metodologia da determinação da massa desta câmara e que, através deste método, importantes inferências poderão ser determinadas, relacionando a massa do ventrículo direito com a sua função, nas diversas afecções que podem acometer esta câmara cardíaca. 


\section{Referências}

1. Foale R, Niho Yannopoulos P, Mckenna W, et al. Echocardiographic measurement of the normal adult right ventricle. Br Heart J 1986; 56: 33-44.

2. Feigenbaum H. Echocardiographic evaluation of cardiac chambers. In: Echocardiography. Pensylvania: Lea \& Febiger, 1994.

3. Dunn FG. Left ventricular hypertrophy in hypertension. N Engl J Med 1999; 340: 1279-80.

4. Savage DD, Drayer JIM, Henry WL. Echocardiographic assessment of cardiac anatomy and function in hypertensive subjects. Circulation 1979; 59: 623-32.

5. Hammond IW, Devereux RB, Alderman MH, Laragh JH. Relation of blood pressure and body build to left ventricular mass in normotensive and hypertensive employed adults. J Am Coll Cardiol 1988; 12: 996-1004.

6. Ganau A, Devereux RB, Roman MJ, Pickering TG, Schnall PL, Laragh JH. Influence of chamber size on left ventricular mass in hypertension. Circulation 1988; 78(suppl II): 519.

7. Safar ME, Toto-Moukonno JJ, Boutheir JA. Arterial dynamics, cardiac hypertrophy, and antihypertensive treatment. Circulation 1987; 75(suppl I): I-156- I-61.

8. Devereux R. Hypertensive cardiac hypertrophy: Pathophysiologic and clinical characteristics. In: Laragh JH. Hypertension: Pathophisiology, Diagnosis and Management. New York: Raven Press, 1990: 359-77.

9. Kauma H, Ikaheimo M, Savolainen MJ, et al. Variants of renin-angiotensin system genes and echocardiographic left ventricular mass. Eur Heart J 1998; 19: 1109-17.

10. Baltatu O, Silva JA, Ganten D, Bader M. The brain renin-angiotensin system modulates angiotensin II induced hypertension and cardiac hypertrophy. Hypertension 2000; 35(part 2): 409-12.

11. Cuspidi C, Sampieri L, Angioni L, et al. Effects of long-term blood pressure control on left and right ventricular structure and function in hypertensive patients. J Cardiovasc Pharmacol 1991; 17(suppl. 2): S517-S18.

12. Russek HI, Zohman BL. The syndrome of Bernheim as a clinical entity. Circulation 1950; 1: 759-65.

13. East T, Bain C. Right ventricular stenosis. Br Heart J 1949; 11: 145-54.

14. Selzer A, Bradley HW, Willett FM. A critical appraisal of the concept of Bernheim’s Syndrome. Am J Med 1955; 18: 567-76.

15. Gottdiener JS, Gay JA, Maron BJ, Fletcher RD. Increased right ventricular wall thickness in left ventricular pressure overload: echocardiographic determination of hypertrophic response of the nonstressed ventricle. J Am Coll Cardiol 1985; 6: 550-5.

16. Nogueira JB, Mourão LM, Morais C, Silva D, Soares-Costa JTS, Costa JNC. Hipertensão arterial e coração direito. Estudo hemodinâmico e ecocardiográfico. Rev Port Cardiol 1991; 10: 837-43.

17. Armour JA, Randall WC. Structural basis for cardiac function. Am J Physiol 1970; 218: 1517-23.

18. Olivari MT, Fiorentini C, Polese A, Guazzi MD. Pulmonary hemodynamics and right ventricular function in hypertension. Circulation 1978; 57: 1185-90.

19. Bloomfield R, Lauson HD, Cournand A, Breed ES, Richards DW. Recording of right heart pressures in normal subjects and in patients with chronic pulmonary disease and various types of cardio-circulatory disease. J Clin Invest 1946; 25 : 639-64.

20. Ramires JAF. Hipertensão arterial sistêmica: estudo em repouso e exercício das alterações hemodinâmicas, da função ventricular e dos fatores que influenciam o débito cardíaco. São Paulo, 1984 (Tese de Livre-Docência, Faculdade de Medicina da Universidade de São Paulo).

21. Lage SG, Ramires JAF, Bellotti G, et al. Análise do comportamento hemodinâmico da circulação pulmonar durante o exercício na hipertensão arterial leve. Arq Bras Cardiol 1983; 41: 85
22. Ratti M, Ramires JAF, Lage SG, et al. O papel do bloqueio beta adrenérgico no comportamento hemodinâmico da circulação pulmonar na hipertensão arterial leve. Estudo em repouso e exercício. Arq Bras Cardiol 1983; 41: 86.

23. Oliveira SF. Hemodinâmica em hipertensão arterial. Análise do comportamento das funções cardíaca e sistêmica nas diferentes fases do exercício. São Paulo, 1985 (Tese de doutorado - Faculdade de Medicina da Universidade de São Paulo).

24. Coelho OR. Comportamento da circulação pulmonar e do ventrículo direito em pacientes portadores de hipertensão arterial sistêmica não complicada. Estudo em repouso e no exercício. Campinas-SP, 1993. (Tese de doutorado - Faculdade de Ciências Médicas da UNICAMP).

25. Amanuma S, Sekiguchi M, Ogasawara S, Honda M, Hosoda S. Biventricular endomyocardial biopsy findings in essential hypertension of graded severity. Postgrad Med J 1994; 70: S67-S71.

26. Prakash R, Lindsay P. Determination of right ventricular wall thickness by echocardiogram. JAMA 1978; 239: 638-40.

27. Prakash R. Determination of right ventricular wall thickness in systole and diastole: Echocardiographic and necropsy correlation in 32 patients. Br Heart J 1978; 40: 1257-61.

28. Nunes BD, Messerli FH, Amodeo C, Garavaglia GE, Scmieder RE, Frolich ED. Biventricular cardiac hypertrophy in essential hypertension. Am Heart J 1987; 114: 813.

29. Cacho A, Prakash R, Sarma R, Kaushik. Usefulness of two-dimensional echocardiography in diagnosing right ventricular hypertrophy. Chest 1983; 84: 154-7.

30. Devereux RB, Gottlieb GJ, Alonso DR. Echocardiographic detection of right ventricular hypertrophy. Circulation 1980; 62(suppl.3): 33.

31. Danchin N. Interventricular septum and right ventricular function. Am Heart J 1994; 129: 1227

32. Noordegraaf AV, Marcus JT, RoseboomB, Postmus PE, Faes TJ, Vries PM. The effect of right ventricular hypertrophy on left ventricular ejection fraction in pulmonary enphysema. Chest 1997; 112: 640-5.

33. Ferlinz J. Right ventricular performance in essential hypertension. Circulation 1980; 61: 156-62

34. Cohn JN, Limas CJ, Guiha MD. Hypertension and the heart. Arch Intern Med 1974; 133: 969-79.

35. Louie EK, Lin SS, Reynertson SI, Brundage BH, Levitsky S, Rich S. Pressure and volume loading of the right ventricle have opposite effects on left ventricular ejection fraction. Circulation 1995; 92: 819-24.

36. Tei C, Dujardin KS. Pressure and volume loading of the right ventricle have opposite effects on left ventricle. Circulation 1996; 94: 2307-8.

37. Habib GB, Mann DL, Zoghbi WA. Normalization of cardiac structure and function after regression of cardiac hypertrophy. Am Heart J 1994; 128: 333-43.

38. Habib GB, Zoghbi WA. Doppler assessment of right ventricular filling dynamics in systemic hypertension: comparison with left ventricular filling. Am Heart J 1992; 124: 1313-9.

39. Aristizabal D, Messerli FH, Frohlich ED. Disparate structural effects on left and right ventricles by angiotensin-converting enzyme inhibitor and calcium antagonist in essential hypertension. Am J Cardiol 1994; 73: 483-7.

40. Saito A, Ueda K, Nakano H. Right ventricular volume determinations by two-dimensional echocardiography. J Cardiogr 1981; 11: 1159-68.

41. Watanabe T, Katsume H, Matsukubo H, Furukawa K, Ijichi H. Estimation of right ventricular volume with two-dimensional echocardiography. Am J Cardiol 1982; 49: 1946-53.

42. Pontes Jr SC. Massa ventricular direita: estudo ecocardiográfico bidimensional. Arq Bras Cardiol 1998; 71: 523-5. 